\title{
La corrupción internacional. Un examen empírico de sus causas
}

\section{María González de Asís*}

\section{Introducción}

-Si los bombres fueran ángeles, no seria necesario ningún gobierno. Si los ángeles tuvieran que gobernar a los hombres, no serian necesarios ni los controles externos ni los internos sobre el gobier. now.

James MADISON, 1790

Aunque la corrupción es un problema antiguo, que ha sido estudiado teóricamente desde Aristóteles, los expertos raramente han analizado empíricamente qué causa la corrupción, a pesar de la vasta incidencia de la comupción en distintos países y en distintos períodos de tiempo. La corrupción existe obviamente en todas las sociedades, pero es más común en unas que en otras, y más común en ciertos períodos de tiempo en la evolución de una sociedad (HuNTNGTON, 1983). Muchos estudios empíricos han explorado el impacto de la comupción, especialmente en el crecimiento económico y en los valores de la sociedad '. La compción ha demostrado ser perjudicial tanto social como económicamente, siempre y donde quiera que ocurra. Para saber cómo combatir la corrupción, es importante analizar los factores que determinan el grado de corrupción en un determinado país. Un estudio teórico y empírico de las causas de la corrupción es, por tanto, esencial para crear un programa efectivo de política pública para combatir la corrupción. Este proyecto explora, teórica y empíricamente, los factores que explican el grado de corrupción política que se percibe en una sociedad y por qué cambia significativamente entre los distintos países.

La corrupción está probablemente ligada a factores económicos, sociales, institucionales y políticos. En países donde hay un lento crecimiento económico, estratificación social y polarización de clases y una alta inestabilidad, la corrupción prevalece. Estas condiciones proporcionan un sistema más articulado a la gente que entra en política para hacer dinero, y conducen, así, a una mayor corrupción política. Este estudio examina el significado de los factores económicos, sociales, institucionales y políticos para explicar la variación de la percepción de corrupción usando medidas agregadas de estos factores de un corte transversal de 53 países. Para observar la variación causal, hago una regresión en la medida de la corrupción percibida en un vector de variables explicativas. Los resultados demuestran cómo el incremento de estabilidad económica y de igualdad social da lugar a una disminución de la comupción. Además, los resultados muestran cómo las débiles estructuras burocráticas y los altos niveles de inestabilidad política contribuyen al aumento de la corrupción.

La siguiente sección incluye un análisis literario de la corrupción. Después, presento un modelo conceptual para explicar cómo las variables económica, política, institucional y social, están teorizadas para fijar el nivel de corrupción. En la sección IV se discuten los datos específicos y las estrategias de estimación usadas en este estudio. Las advertencias y limitaciones están en la sección V. Los hallazgos y los resultados de la estimación se describen en la sección VI. Finalmente, se exponen las conclusiones orientadas a las implicaciones políticas.

\section{Análisis de la literatura}

El estudio de las causas de la corrupción tiene una larga historia, que se remonta por lo menos hasta la literatura basada en 
la búsqueda de ganancias (rent seeking) y la corrupción (ver BhaGWaTI, 1982; KrUEGer, 1974; Rose ACKERMan, 1978; TUllock, 1967; y AMARO REYES, 1983). Sin embargo, el trabajo empírico relacionado con las causas sociales y políticas de la comupción ha sido bastante limitado, debido, en parte, a la eficiencia de las instituciones gubernamentales y a que la corrupción en particular no puede ser fácilmente cuantificada.

La discusión sobre la corrupción se ha orientado en primer lugar a las constantes consecuencias negativas de la corrupción. JOHNSTON (1986), ACKERMAN (1996), MAURO (1996) y otros han mostrado que la corrupción es perjudicial para un país, tanto social como económicamente. La corrupción conduce a una ineficacia y a un derroche económico debido a su impacto en la asignación de fondos, en la producción y en el consumo (GOULD y AMARO REYES, 1983).

El renovado interés en el tema de la corrupción política ha llevado recientemente, a un número de investigadores a tratar de cuantificar el alcance en el que la compción penetra en las interacciones económicas, sociales y políticas (SHIEIFER, 1993; Evans, 1996). Lo hacen utilizando índices del nivel de corrupción política elaborados por agencias privadas de evaluación. Estos índices están típicamente basados en la información proporcionada por consultores situados en varios países. Los índices proporcionan una riqueza de información que ha permitido a los investigadores obtener un número interesante de resultados, a pesar de la naturaleza limitada de los datos.

Hasta hoy la tradición académica ha sido la de practicar una regresión de un representante de los resultados de índices de corrupción. El objetivo era descubrir las consecuencias negativas de la corrupción en el desarrollo de un país, determinar específicamente la correlación entre el nivel de corrupción en un país y el crecimiento económico (Mauro, 1996).

Por último, la tradición académica se ha dedicado más al estudio de la corrupción desde la perspectiva de la rent seeking asociada a la corrupción política, ya que algunas personas han tratado de entrar en política para hacer dinero fácil, aprovechándose de las situaciones institucionales, económicas y sociales de su país. Por lo tanto, las causas económicas, sociales e institucionales de la rent seeking han sido profundamente analizadas como potenciales causas significativas de la corrupción. La literatura original de la rent seeking enfatizó las restricciones del comercio como el principal ejemplo de fuentes de ingresos inducidas por el gobierno (KRUEGER, 1974). Estudios posteriores relacionan la corrupción con factores económicos y financieros. Por ejemplo, ADES y Di TELLA (1994) han descubierto que un alto grado de apertura de la economía, medida en base a la suma de importaciones y exportaciones como parte del PIB, está significativamente asociado a una menor corrupción y a una menor rent seeking. Además, las subvenciones del gobicr. no, incluidos los gastos fiscales, pueden constituir fuentes de rentas, como sostuvo ClemenTS (1995).

No se espera que un número adicional de fuentes de rentas sea debido a la política gubernamental. Los intentos de evaluar los efectos de ciertos aspectos de la política gubernamental en la corrupción necesitan tener en cuenta estos otros factores. Por ejemplo, la dotación de recursos naturales constituye un ejemplo de libro de las fuentes de las rentas, dado que pueden venderse típicamente a un precio que excede con mucho su coste de extracción. SaCHS y WarNer (1995) argumentan que es más probable que las economías con vastos recursos naturales puedan estar sometidas a un comportamiento externo de rent seeking de lo que lo están las economías pobres en recursos. En concreto, han descubierto que la fracción de las exportaciones de producto primario sobre las exportaciones totales está asociada a los índices de eficiencia burocrática, aunque no a los niveles convencionales de significación estadística.

Los bajos salarios de la función pública con relación a los salarios del sector privado o PIB per capita, son también una fuente potencial de (un nivel bajo de) corrupción, como lo predijeron KRaAY y Van RJCKEGHEm (1995) y HaQue y SAHAY (1996). Cuando los salarios de la función pública son demasiado bajos, los funcionarios pueden ser más propensos a usar sus posiciones para aceptar sobornos como una forma de cuadrar sus gastos con sus ingresos. En la mayoría de los casos, el coste esperado de ser descubiertos y despedidos es generalmente bajo (Departamento de Hacienda, 1995).

Hoy, es más probable que el debate sobre las causas de la corrupción se vea desde un contexto distinto del de rent seeking con múltiples aproximaciones: económica, institucional, social y política. Estas aproximaciones muestran un tratamiento teórico más completo de las causas de la corrupción y permite un diagnóstico más efectivo de este corrosivo fenómeno.

Organizaciones internacionales como por ejemplo el Consejo de Europa (CE), las Naciones Unidas (ONU), la Organización de Estados Americanos (OEA y Organizaciones No Gubernamentales (ONGs) como Transparencia Intemacional (TI), han dedicado sus esfuerzos a subrayar las causas teóricas y las consecuencias de la corrupción, así como a generar directrices para futuros investigadores de esta materia, tales como las del Libro de Fuentes de TI (1996).

Este proyecto intenta presentar, de manera unificada, tomando tanto una visión teórica como práctica, las causas más importantes de la corrupción halladas por expertos en el pasado. Para que la comupción exista, tiene que haber oportunidades para que se desarrolle un mercado negro, y estas oportunidades han sido estudiadas por los expertos desde dos perspectivas diferentes: una alude a características individuales, mientras que la otra se centra en influencias estructurales. En este proyectō, 
las raices subyacentes de la corrupción incluyen influencias económicas, sociales, institucionales, políticas y educacionales, que explican, en un contexto empírico, la variación del nivel de comupción en los distintos países. El entramado presentado en este trabajo no es exhaustivo y no pretende determinar todas las causas de la corrupción. Sin embargo, explora el tema de la corrupción desde las perspectivas combinadas de la ciencia económica y política para desplegar un acercamiento integrado que permita el desarrollo de una política pública coherente.

El modelo conceptual presentado en la siguiente sección identifica estas causas y sugiere hipótesis sobre la relación positiva o negativa entre la inestabilidad económica, la igualdad social, la burocracia eficiente, la estabilidad política y el nivel de educación y la corrupción.

\section{Modelo conceptual de la corrupción política}

La gráfica 1 muestra el modelo conceptual que desarrollo en la siguiente sección. El objetivo de.este modelo es determinar hasta qué punto la inestabilidad económica, la desigualdad social, la burocracia ineficiente y la inestabilidad política, explican la variación en la percepción de la corrupción en los distintos países. La teoría trata de explicar objetivamente las relaciones entre las variables presentadas en el modelo y la corrupción.

Primeramente, es probable que haya una relación negativa entre la corrupción y la inestabilidad económica, donde la estabilidad económica de un país depende de la libertad económica, del indice de crecimiento del PIB y de la inflación anual. Concretamente, un bajo nivel de libertad económica, un bajo índice de crecimiento del PIB y una alta inflación anual, darán, probablemente, como resultado un alto nivel de corrupción. Esto se debe a que la corrupción se produce ampliamente, si la economía de un país ofrece grandes posibilidades de prosperar cuando los funcionarios públicos y políticos comuptos son vulnerables a una abusiva manipulación del negocio en economías dominadas por un pequeño número de empresas. En economías donde hay un alto grado de interferencia del gobierno, existe también un incentivo para la corrupción, como también lo hay en economías donde las empresas nacionales están protegidas de la competitividad extranjera gracias a altas tarifas y donde los ciudadanos se enfrentan a una alta inflación.

También observaremos probablemente una relación negativa entre la corrupción y la igualdad social. Cuando hay un bajo nivel de igualdad social, que se mide usando el nivel de educación como un equivalente, la corrupción aumenta. Cuando hay un alto nivel de no-escolarización, la desigualdad social aumenta y así también el incentivo para la comupción. Es más probable que las personas que han tenido una educación menor formen parte de un sistema corrupto. Las personas no escolarizadas pueden carecer del conocimiento básico de los valores sociales, morales y éticos. Más aún, las diferencias en el nivel de educación en una sociedad aumentan la desigualdad social. A medida que la desigualdad social aumenta, así también aumentan las oportunidades para los mercados negros, porque los ciudadanos buscan una forma más rápida de conseguir más propiedades, más bienes y más riqueza.

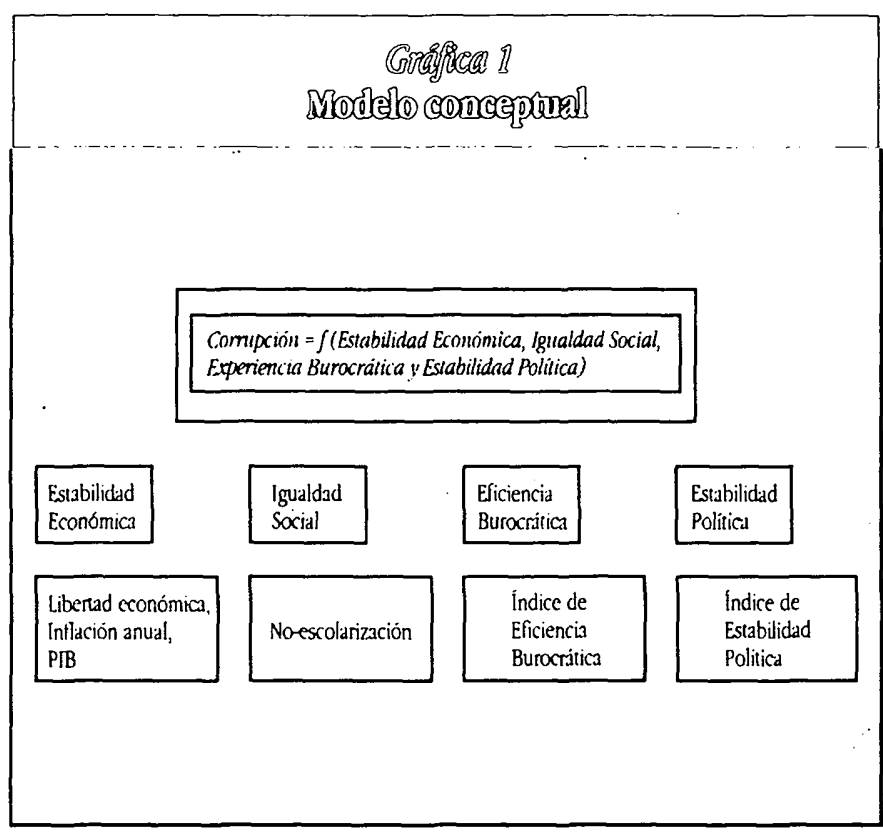

No hay una medida absoluta para medir la igualdad social en los distintos países. El coeficiente Gini es el indicador más usado para medir la igualdad de ingresos. Sin embargo, los datos sobre la distribución de ingresos no son actualmente muy completos, por lo que la educación se usará como un equivalente para medir la igualdad social.

Desde el punto de vista institucional, también es probable que haya una relación negativa entre la corrupción y la eficiencia de las estructuras burocráticas. Cuando la eficiencia burocrática es débil y existe una excesiva burocracia, se crea una atmósfera fértil para el desarrollo del comportamiento corrupto.

Finalmente, también se espera una relación negativa entre la corrupción y la estabilidad política. Cuando hay un bajo nivel de estabilidad política, la corrupción aumenta. El cambio institucional, el cambio social, que la oposición tome el poder y el terrorismo, son variables que ofrecen oportunidades de muchas clases para que se desarrolle un mercado negro de corrupción. La corrupción puede ser más dañina y por tanto ser denunciada como un problema más serio en países políticamente inesta- 
bles, Mauro (1995). Shleifer y Vishmy (1993) argumentan que los países con gobiernos débiles (y por tanto inestables) experimentarán un tipo de corrupción muy perjudicial.

El patrón seguido por las variables de la Gráfica 1 forma el entramado que describe y motiva la estrategia de estimación discutida en la siguiente sección. Pruebo cuatro hipótesis específicas en función del objetivo de este estudio, que es determinar hasta qué punto la inestabilidad económica, la igualdad social, las estructuras burocráticas y la estabilidad política explican la variación en la percepción pública de la corrupción en los distintos países:

Hipótesis 1: Estabilidad Económica: cuando hay una baja estabilidad económica, existen incentivos para desarrollar un comportamiento corrupto.

Hipótesis 2: Igualdad Social: cuando existe un bajo nivel de educación, la igualdad social disminuye y, por tanto, la corrupción aumenta.

Hipótesis 3: Eficiencia Burocrática: cuando las estructuras burocráticas son débiles, se crea una atmósfera apropiada para el desarrollo de un comportamiento corrupto.

Hipótesis 4: Estabilidad politica: Cuando hay un bajo nivel de estabilidad política en un país, es más probable que se dé la corrupción.

En la siguiente sección, presento la estrategia de estimación usada para probar las hipótesis expuestas en el modelo conceptual.

\section{Descripción de los datos y estrategia de estimación}

\section{Descripción de los datos}

La variable dependiente en este estudio, la corrupción, es una medida gradual que representa el grado de corrupción política de una sociedad. El grado de corrupción está basado en el nivel de corrupción en un país percibido por los empleados de empresas multinacionales e institucionales. Existen dos índices de corrupción, uno para 1995 y otro para 1996. Estos índices fueron preparados por Transparencia Internacional (TT), una organización no lucrativa dedicada al el estudio de la corrupción. Ambos índices fueron recogidos de varios estudios de TI de hombres de negocios y periodistas. Los índices incluyen los estudios de -The World Competitive Report del The Institute for Manage- ment Development en Lausana (1992-1994), la empresa The Politic and Economic Risk Consultancy Lid., en Hong Kong (19921994) y finalmente, un estudio de 1980 de hombres de negocios que operaban en Nueva York. El índice de 1996 es un listado más completo de 53 países, mientras que el índice de 1995 sólo lista 43 países. Por lo tanto, en este estudio se usa el índice de 1996. Una puntuación de 10 indica un país perfectamente "limpion, mientras que un 0 se refiere a un país donde las transacciones políticas están totalmente empapadas por la compción, que abarca una inmensa suma de comisiones, extorsión, fraude y similares. (Ver Apéndice de tabla 1 para los datos en todas las variables usadas en este estudio).

El primer bloque de variables independientes en el modelo mide la estabilidad económica en una sociedad. Existen tres medidas utilizadas para reflejar los niveles de estabilidad económica. El primero es el Índice de Libertad Económica, que mide el nivel de libertad económica en países de todo el mundo. Para ello, se ha establecido este bloque de criterios económicos objetivos por la Fundación Heritage en colaboración con el Banco Mundial para clasificar 150 países y crear un índice que mida la libertad económica en los distintos países (Holmes, JOHNSON y KIRKPATRICK, 1997). Aunque existen muchas teorías sobre los orígenes y las causas del desarrollo económico, los hallazgos del estudio sobre el desarrollo económico realizado por la Fundación Heritage son concluyentes: aquellos países con mayor libertad económica tienen índices más altos de desarrollo económico que aquellos que tienen menor libertad económica. El Índice de Libertad Económica mide cómo puntúan esos países en una lista de diez factores económicos ${ }^{2}$. Cuanto mayor es la puntuación, mayor es la interferencia del gobiemo en la economía y, por tanto, menor libertad económica hay. Un país con una nota de 1.00 a 1.99 se considera libre. Un país con una nota de 2.00 a 2.99 es considerado mayoritariamente libre, y un país con una nota de más de 3.00 es considerado mayoritariamente no-libre.

La segunda medida de la estabilidad económica es el íñdice de inflación anual en el período de 1984 a 1994. Esto refleja el cambio de precio para todos los bienes y servicios producidos en una economía. A mayor puntuación, mayor es la inflación anual. Los datos fueron proporcionados por el Banco Mundial, Informe del Desarrollo Mundial, 1996.

La última medida de la estabilidad económica es el índice de crecimiento del PIB, que capta el nivel de desarrollo económico en un país, entre 1983 y 1993. A mayor puntuación, mayor es el índice de crecimiento del PIB. Los datos provienen del Banco Mundial, Informe del Desarrollo Mundial, 1996.

El siguiente determinante hipotético de la corrupción en el modelo conceptual es la igualdad social. La educación se ha utilizado como un equivalente de la igualdad social en el modelo. La educación se mide por el porcentaje de la población no escolarizada, en ${ }^{-1985}$. Se cifra el alto nivel de educación en 
un porcentaje de la población no escolarizada o inferior. Se considera bajo nivel de educación a la puntuación del $4 \%$ o más. Estos datos son del Informe del Desarrollo Mundial, 1996, del Banco Mundial. Los datos usados son de 1985 y 1996. El índice de 1985 es el más completo de los índices. Expresa el nivel de cultura de personas que fueron educadas hace diez años y que pueden formar parte de una práctica corrupta por sus posiciones, u oponerse a la práctica en el sistema social.

El tercer bloque de variables independientes en el modelo mide la eficiencia burocrática. Representan la fuerza de las estructuras burocráticas en el período de 1980 a 1983. El índice de eficiencia burocrática es la media de los índices de lo judicial, del exceso de burocracia y de la compción. Una puntuación de 10 en los índices de eficiencia burocrática y de exceso de burocracia, representa el mayor nivel de eficiencia burocrática; una puntuación de 4 representa una baja eficiencia burocrática. Los datos son de Mauro (1983). El índice de la burocracia de 1983 es el índice más reciente. Las estructuras burocráticas no son propensas a grandes cambios en cortos períodos de tiempo en un mismo país. Con la excepción de Méjico y Argentina, que están sufriendo enormes reformas en la Administración, el resto de los países no han experimentado importantes reformas burocráticas en diez años. Por lo tanto, el índice de 1983 debería captar la eficiencia burocrática diferencial en los distintos países.

La cuarta variable independiente para medir la corrupción corresponde al nivel de estabilidad política en un país. El índice de estabilidad política es la media de seis índices individuales: el Cambio Institucional; el Cambio Social; que la Oposición tome el poder; la Estabilidad Laboral; los Países Vecinos; y el Terrorismo, lo que representa el nivel de estabilidad políica en un país para 1983. Este índice fue támbién creado por MAURO (1983). A mayor puntuación, mayor estabilidad política.

Las estadísticas descriptivas de las variables dependientes $\mathrm{e}$ independientes están en la Tabla 1. En la siguiente sección discuto la estrategia de estimación utilizada en este estudio.

\section{Estrategia de estimación}

La estimación de los parámetros del modelo escogido se hará en SPSS. Dado que este modelo está basado en modelos de regresión parcial con múltiples variables explicativas, el método estadístico utilizado es el de cuadrados menos comunes (ordinary least squares). El objetivo es medir la relación entre la variable dependiente, la corrupción y el conjunto de variables explicativas descritas en el modelo.

Comupción $=f$ (Estabilidad Económica, Desigualdad Social, Insuficiencia Burocrática, Estabilidad Política)
En la estimación del modelo conceptual, algunas de las va-riables independientes podrían ser altamente correlacionadas. Esto significa que se espera algún problema de multicolinearidad entre las variables del modelo. Éste es un problema que puede ser detectado fácilmente utilizando algunos coeficientes de regresión parcial entre las variables independientes. Una opción para evitar la multicolinearidad es hacer una estimación de una forma reducida del presente modelo, como, por ejemplo, la Comupción como una función solamente del crecimiento del PIB y de las estructuras burocráticas. Sin embargo, eso puede dar lugar a una propensión a la especificación. Otra posibilidad es omitir una de las variables que pueda ser correlacionada, y añadir algunas otras variables.

Un problema adicional es la heterodasticidad (beteroscedasticity). Esto es, es posible encontrar considerables varianzas desiguales en la variable dependiente para los mismos valores de la variable independiente. El problema puede detectarse representando gráficamente las variables y examinando la figura de lo que resulte.

Los problemas en la estrategia del modelo conceptual no son las únicas limitaciones a tener en cuenta en este proyecto. Existen ciertas advertencias y consideraciones teóricas que se discutirán en la próxima sección.

\section{Advertencias y limitaciones}

Es importante reconocer que así como en el modelo uso la percepción de la corrupción política como la variable dependiente, los estimadores que son tomados en consideración en el modelo conceptual afectan, presumiblemente, a la corrupción actual. Debido a esto, lo que el modelo hace realmente es medir las relaciones entre los factores y las percepciones, con la asunción tácita de que las percepciones son una medida objetiva de la realidad. Por lo tanto, tenemos que asumir que la percepción de la corrupción equivale a la corrupción. El problema de esta asunción es que las medidas estádísticas basadas en percepciones entrañan cierto riesgo, dado que las percepciones de las personas no siempre coinciden con la realidad, y esto tiene que tomarse en cuenta para realizar un análisis ajustado del modelo:

Otro problema a tener en cuenta es la posible causalidad inversa del modelo. Esto signifiea que la comupción puede estar no sólo causada por factores económicos, sociales, burocráticos, políticos y educacionales, sino que puede producir consecuencias económicas, sociales, institucionales, políticas y educacionales. Éste es un tema para futuras investigaciones. 
Tatolos II

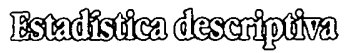

\begin{tabular}{|c|c|c|c|c|}
\hline VARUABLES & DEFHILNSN & MEDIA & MADIARIA & 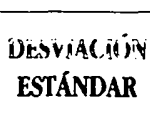 \\
\hline $\begin{array}{l}\text { INDICE DE } \\
\text { CORRUPCIÓN } \\
1996\end{array}$ & $\begin{array}{l}\text { Grado de comupción política en un país } \\
10=\text { país limpio } \\
0=\text { comupto al máximo. }\end{array}$ & 5.1835 & 4.9950 & 2.6446 \\
\hline $\begin{array}{l}\text { ÍNDICE DE LIBERTAD } \\
\text { ECONỌMICA } 1997\end{array}$ & $\begin{array}{l}\text { Nivel de libertad económica en un país. A mayor puntuación, mayor } \\
\text { interferencia del gobiemo en la economía y, por lo tanto, menor libernad } \\
\text { económica. Un pais con una puntuación de } 1.00 \text { a } 1.99 \text { se considera } \\
\text { libre. Un país con una puntuación de } 2.00 \text { a } 2.99 \text { se considera } \\
\text { mayoritariamente libre y un país con una puntuación mayor de } 3.00 \text { se } \\
\text { considera mayoritariamente no-libre. }\end{array}$ & 2.61 & 2.50 & 0.72 \\
\hline $\begin{array}{l}\text { INFLACIÓN ANUAL } \\
\text { 1984-1994 }\end{array}$ & $\begin{array}{l}\text { Cambio en el precio de todos los bienes y servicios producidos en una } \\
\text { economía. }\end{array}$ & 21.0612 & 7.9000 & 47.5459 \\
\hline $\begin{array}{l}\text { NO-ESCOLARIZACIÓN } \\
1995\end{array}$ & $\begin{array}{l}\% \text { de la población no-escolarizada. Un uno por ciento o menos se } \\
\text { considera un alto nivel de escolarización. Un cuatro por ciento o más se } \\
\text { considera un bajo nivel de escolarización. }\end{array}$ & 19.1569 & 9.8000 & 22.3283 \\
\hline $\begin{array}{l}\text { EFICIENCIA } \\
\text { BUROCRÁTICA } \\
1980-1983\end{array}$ & $\begin{array}{l}\text { Fuerza de las estructuras burocráticas en el periodo } 1980 \text { y } 1983 \text {. Una } \\
\text { puntuación de } 10 \text { representa el nivel más alto de eficiencia burocrática. } \\
\text { Una punnaación de } 4 \text { representa una baja eficiencia burocrática. }\end{array}$ & 7.0617 & 6.9200 & 2.1857 \\
\hline $\begin{array}{l}\text { ESTABILIDAD } \\
\text { POLITICA }\end{array}$ & $\begin{array}{l}\text { Nivel de estabilidad política en un país en 1995. La media de seis índices } \\
\text { individuales: Cambios Institucionales; Cambios Sociales; Toma de Poder } \\
\text { por pante de la Oposición; Estabilidad Laboral; Países Vecinos y } \\
\text { Terrorismo. Una puntuación inferior a } 7 \text { se considera como un nivel de } \\
\text { estabilidad política muy bajo en un país. Una puntuación mayor de } 9 \text { se } \\
\text { considera un alto nivel de estabilidad política. }\end{array}$ & 7.7690 & 7.8500 & 1.2241 \\
\hline
\end{tabular}

\section{Hallazgos}

\section{Distribución variable}

La distribución de los valores de la corrupción está. representada en la Gráfica 2. El eje horizontal representa todos loş valores posibles de la corrupción en los distintos países y el eje vertical representa la probabilidad de que esos valores ocurran. Podemos observar una distribución casi normal con algunos valores extremos a lo largo del índice de Corrupción de 1996 (los valores que quedan fuera de la muestra, por ser extremos de la misma se concentran en 9-9.5). Los altos valores extremos corresponden a Dinamarca, Finlandia y Nueva Zelanda. Las puntuaciones del índice de corrupción están muy agrupadas alrededor de la media en un patrón bimodal.

El Índice de Libertad Económica está representado en la Gráfica 3. Podemos también observar una distribución casi nor- mal en este diagrama, donde el eje horizontal representa todos los valores posibles de la libertad económica en los diferentes países y el eje vertical representa la probabilidad de que esos valores se produzcan. Hay representado un valor extremo que corresponde a Corea (con una libertad económica muy limitada) que hace que las puntuaciones en la libertad económica no estén exactamente agrupadas alrededor de la media.

El índice de crecimiento del PIB está representado en.la Gráfica 4. La distribución es normal. El eje horizontal representa todos los valores posibles del índice de crecimiento del PIB en los distintos países y el eje vertical representa la probabilidad de que se den esos valores. Otra vez, parece que existe un alto valor extremo correspondiente a Corea, que ha tenido un crecimiento económico extremadamente alto en las últimas décadas.

En la Gráfica 5, la distribución de la inflación no claramente es normal. El eje horizontal representa todos los valores posibles de la inflación anual en los paises y el eje vertical representa la probabilidad de que se den esos valores. La mayoría de los países tienen niveles razonahlemente bajos de inflación, pero exis- 

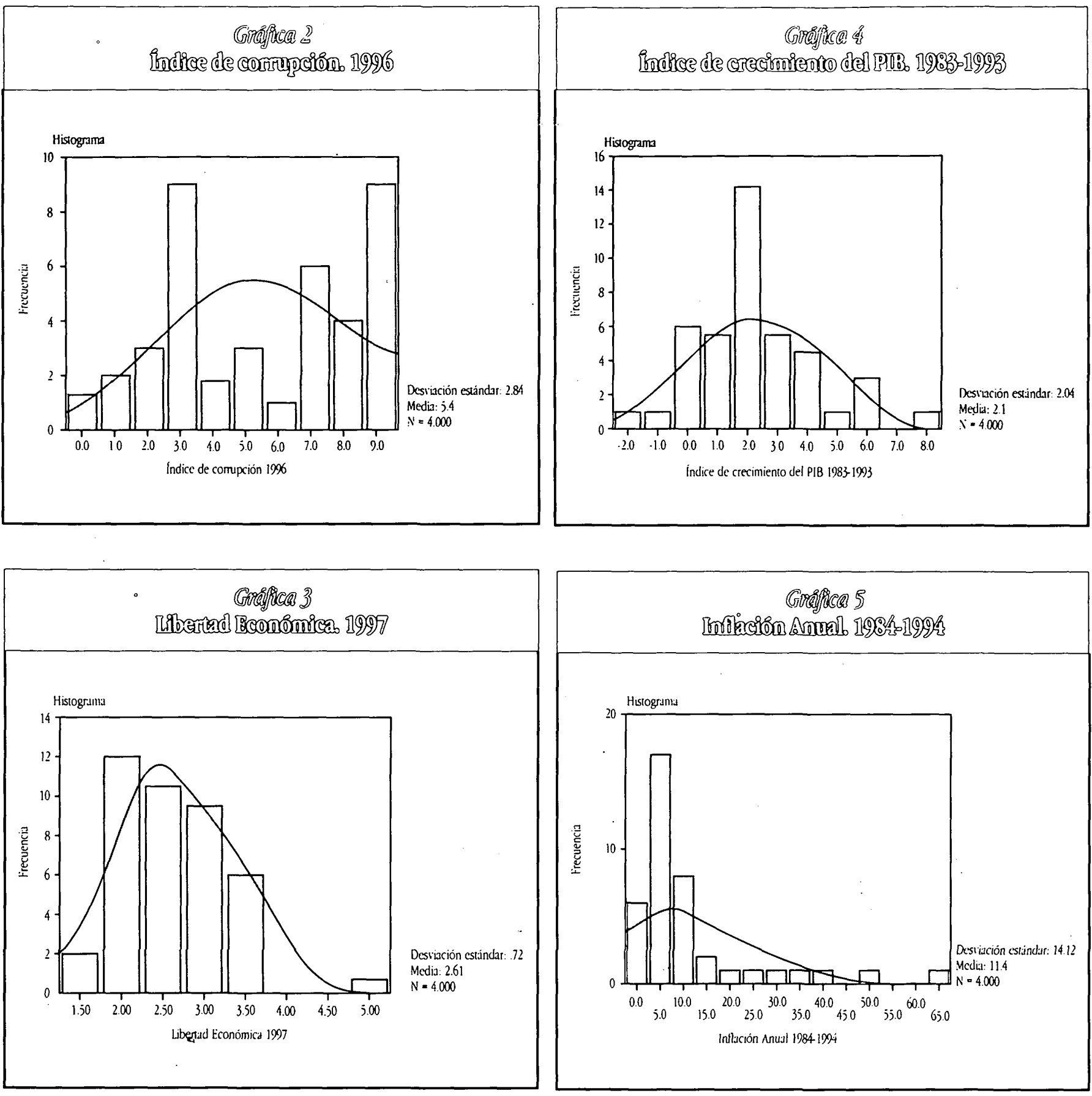

ten países que tienen niveles muy altos de inflación, como por ejemplo Ecuador, Turquía y Venezuela.

La no-escolarización en la Gráfica 6, también tiende a agruparse alrededor de los valores bajos, aunque hay pocos países con un valor alto. El eje horizontal representa todos los valores posibles para la no-escolarización en los diferentes países y el eje vertical representa la probabilidad de que ocurran esos valores. Los valores extremos de 70 y 80 pertenecen a Nigeria y Pakistán.

En la Gráfica 7 podemos observar una distribución normal de la eficiencia burocrática. El eje horizontal representa todos los va-

lores posibles de eficiencia burocrática y el eje vertical representa la probabilidad de que se den esos valores. La puntuación en la Eficiencia Burocrática se agnipa en torno a la media en un patrón casi simétrico y unimodal de curva de campana, o de curva normal, con la media, la mediana y la moda aproximadamente iguales. Los paises con un alto grado de eficiencia burocrática incluyen a Nueva Zelanda y Noruega y los países con un bajo nivel incluyen a Méjico y Nigeria.

Finalmente, en la Gráfica 8 podemos. observar una distribución normal de la estabilidad política. El eje horizontal repre- 

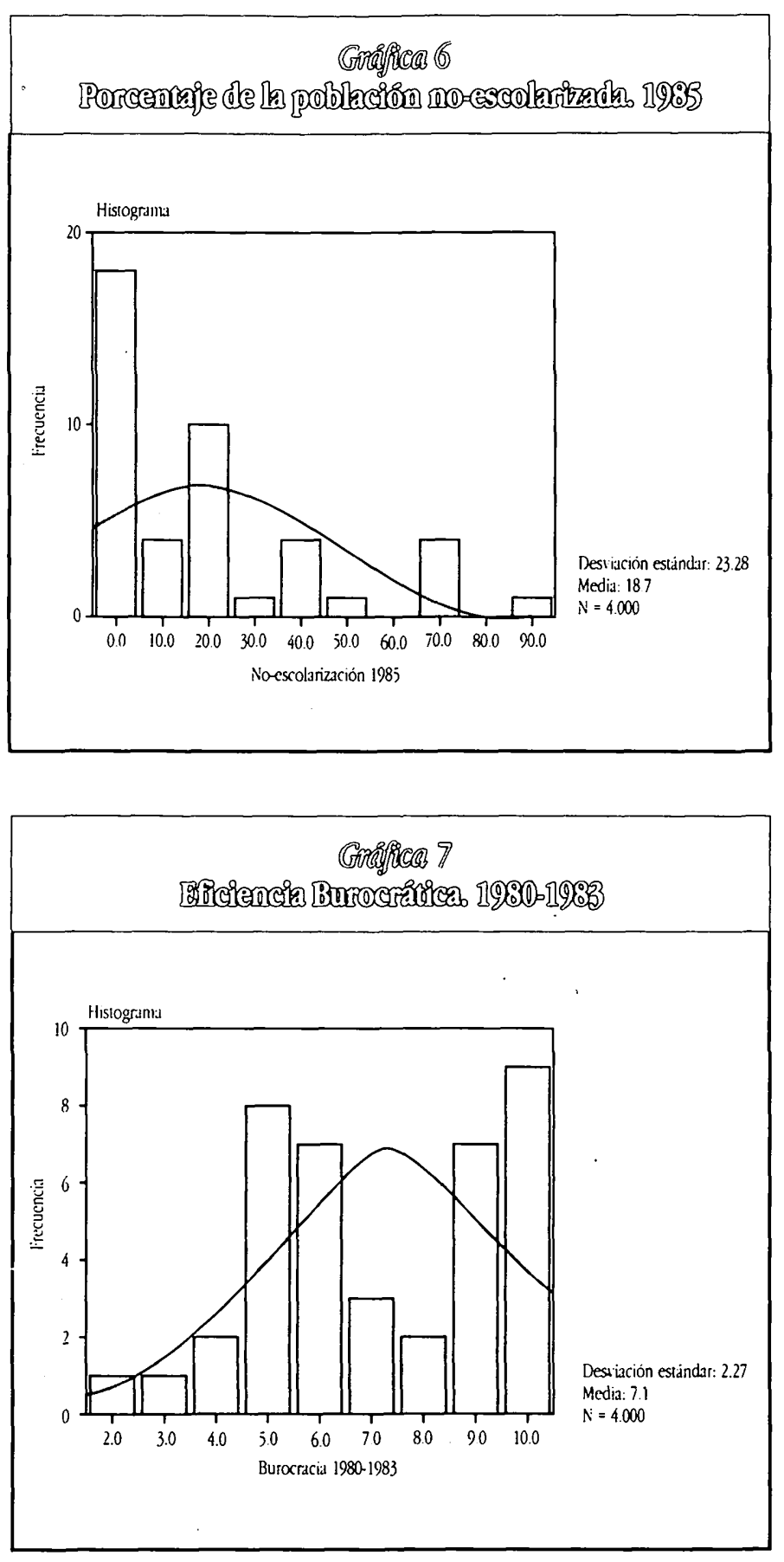

senta todos los posibles valores de la estabilidad política en los diferentes países y el eje vertical representa la probabilidad de que ocurran esos valores. Los paísés con un alto valor incluyen a Suiza y Hong Kong y los países con ùn. bajo valor incluyen a Sudáfrica y Bangladesh.

\section{Resultados de la estimación}

En esta sección se muestran los resultados de dos modelos de regresión. Antes de analizar ambos modelos, se han calcula-

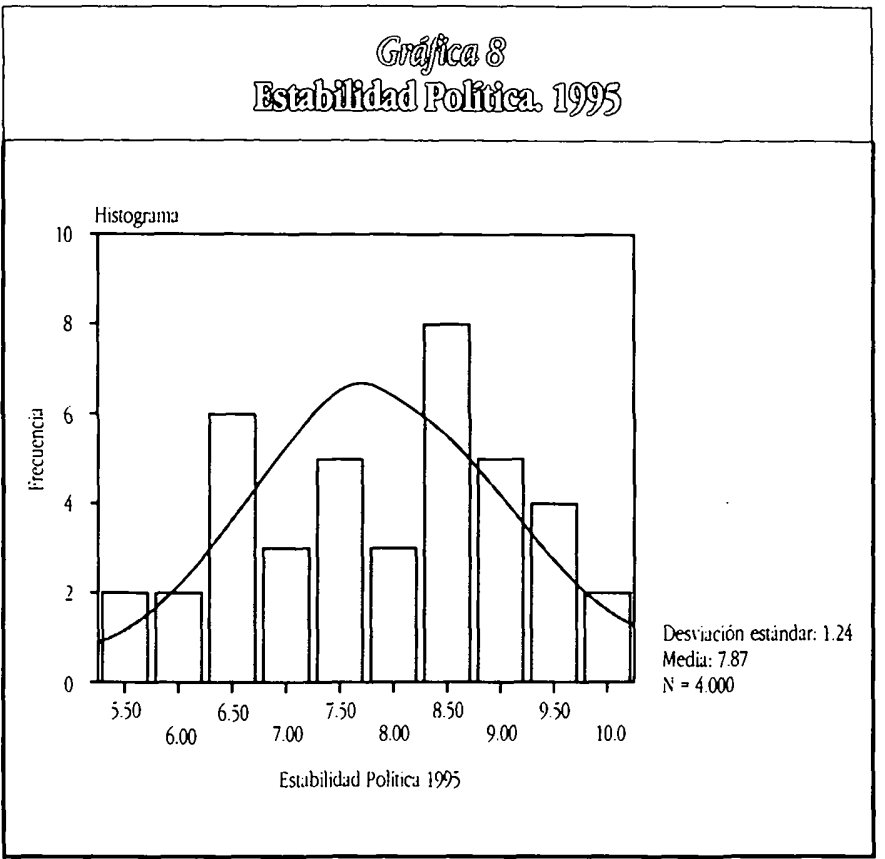

do los coeficientes de correlación entre las variables dependientes y las variables independientes, para ilustrar la relación entre las variables basadas en los datos (Apéndice Tabla 2). Una de las características más sorprendentes del bloque de datos es la fuerte asociación entre la eficiencia burocrática y la estabilidad política. El coeficiente de correlación simple entre el índice de eficiencia burocrática y el índice de estabilidad política es de 0.713 .

A continuación, se generaron histogramas de puntos individuales entre las variables independientes y la comupción. La Gráfica 9 muestra una relación potencialmente negativa entre el índice de la corrupción y la Libertad Económica, que sugieren que a medida que la libertad económica disminuye, aumenta la corrupción.

Aquellos países con una alta puntuación tienen una mayor interferencia del gobierno en la economía y, por tanto, menor libertad económica, y mayor corrupción. Como señala Steve $\mathrm{H}$. HANKE (1996), para que la corrupción exista debe haber oportunidades para llevarla a cabo. Estas oportunidades están en función del tamaño y del poder. del Estado. Cuanto mayor sea el papel del Estado en la economía, mayor será el número de oportunidades para obtener ganancias sobornando a los funcionarios. Los datos apoyan esa lógica. Hay aparentemente menos corrupción en países donde el control del Estado es menor y la libertad económica es mayor. Por ejemplo, Nueva Zelanda tiene un bajo nivel de corrupción equivalente a 9.56 y es un país con una gran libertad económica, del 1.75 .

El histograma de la Gráfica 10 muestra una relación potencialmente positiva entre el índice de comupción y el PIB, de forma que a medida que el PIB crece, el índice de comupción aumenta, lo cual significa que la corrupción desciende. Algunos auto 

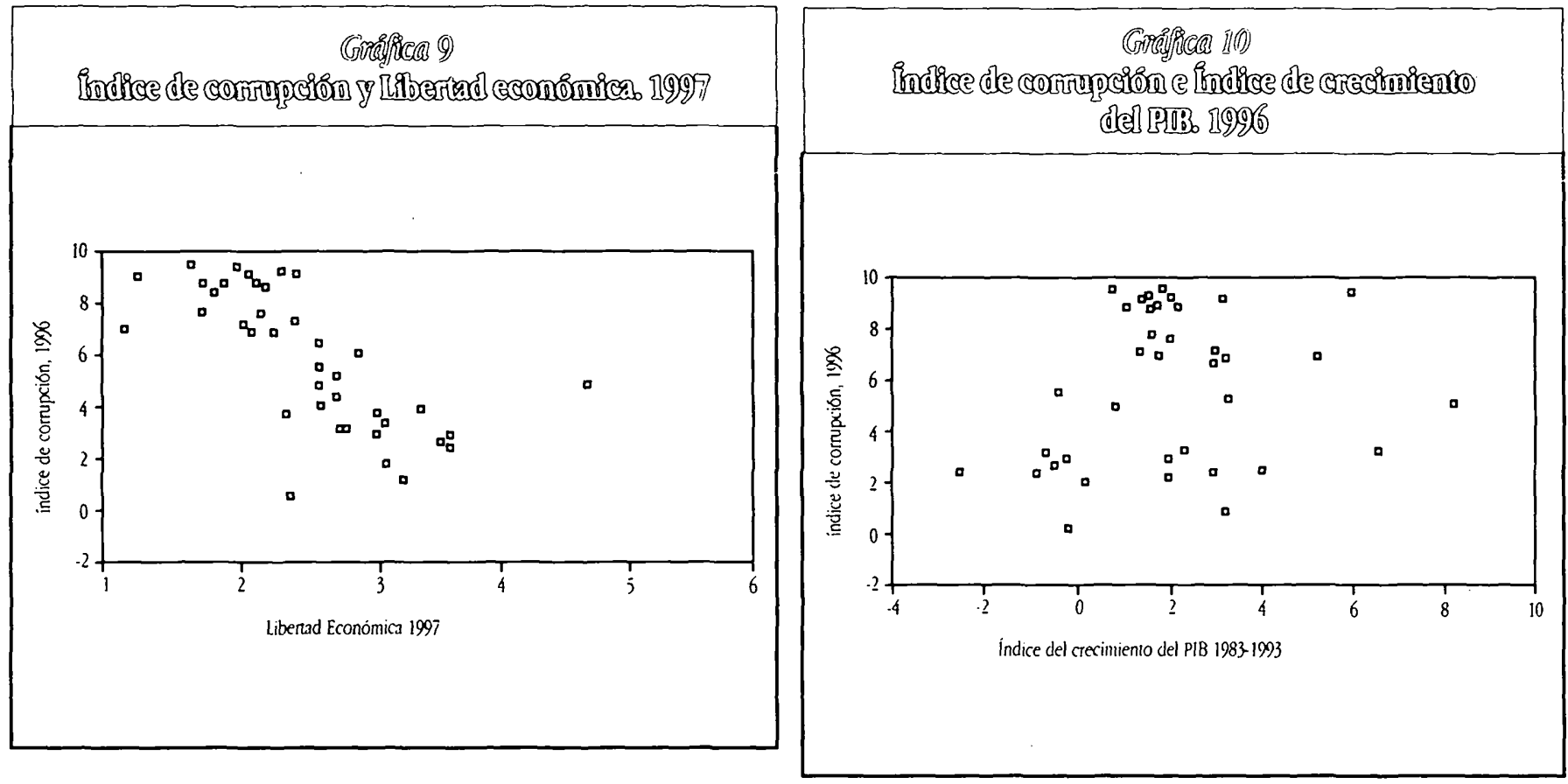

res han sugerido que la corrupción podría aumentar el crecimiento económico, porque las prácticas corruptas permitirían a los individuos evitar el retraso burocrático (LEFF, 1964; HUNTING. TON, 1968). Sin embargo, otro trabajo empírico (MAURO, 1996) sostiene que la corrupción disminuye la inversión privada, reduciendo así el crecimiento económico. Los datos indican aquí una causalidad inversa: el crecimiento económico hace disminuir la corrupción. En países como Indonesia, donde las regulaciones burocráticas son engorrosas, la corrupción puede ser beneficiosa a corto plazo para el crecimiento económico, que es también muy alto (4.2). Sin embargo, en países donde las instituciones gubernamentales son más eficientes, como Singapur, el crecimiento económico (6.1) conduce a una menor corrupción (8.8).

La Gráfica 11 muestra una relación potencialmente positiva entre la corrupción y la inflación anual, y la Gráfica 12 muestra una relación potencialmente negativa entre la comupción (según aumenta la puntuación, disminuye la compción) y la no-escolarización (según aumenta la puntuación, hay menos educación). En países donde 'el porcentaje de la población no escolarizada en 1985 es superior a cuatro, como por ejemplo Nigeria, la corrupción es alta (0.69).

La Gráfica 13 muestra una relación potencialmente positiva entre el índice de corrupción y la eficiencia de la burocracia, lo que significa que a medida que aumenta la eficiencia burncrática, la corrupción decrece. Los países con mejores instituciones y un sistema judictal más eficiente tienen un menor nivel de corrupción, comấ por ejemplo Nueva Zelanda, con un nivel de

estructuras burocráticas de 10 y un índice de corrupción de 9.43 (muy poca corrupción).

Finalmente, la Gráfica 14 muestra una relación potencialmente positiva entre el índice de corrupción y la estabilidad política. Así, a medida que aumenta la estabilidad política, la corrupción parece decrecer. Dinamarca (9.33), Nueva Zelanda (9.43) y Canadá (8.96) son ejemplos de países con un bajo nivel de corrupción y altos niveles de estabilidad políica.

Este estudio presenta dos modelos de regresión (Tabla 2). Estos pueden analizarse según los resultados esperados incluidos en el modelo conceptual. En el Modelo I, se tomaron como variables continuas la Eficiencia Burocrática, la Libertad Económica, el PIB, la Inflación, la No-Escolarización y la Estabilidad Política. Dos variables simuladas (variables dummy) para la localización geográfica, OCDE y Asia oriental, se incluyeron como variables explicativas en el modelo. Esas variables simuladas se añadieron después de observar la posible relación entre los países con una libertad económica menor y un sistema político inestable y corrupción. La asociación se mantenía teóricamente dado que, en general, los países con una menor libertad econónica y un sistema político inestable son parte de la región de Asia oriental. Por lo tanto, esperaba encontrar niveles más altos de corrupción que en países donde hay una mayor libertad económica y una mayor estabilidad política, como es el caso de los países de la OCDE. Sin embargo, los resultados empíricos muestran que no hay una relación significativa entre el hecho de que un país pertenezca a Asia oriental o la OCDE, y la corrupción. 

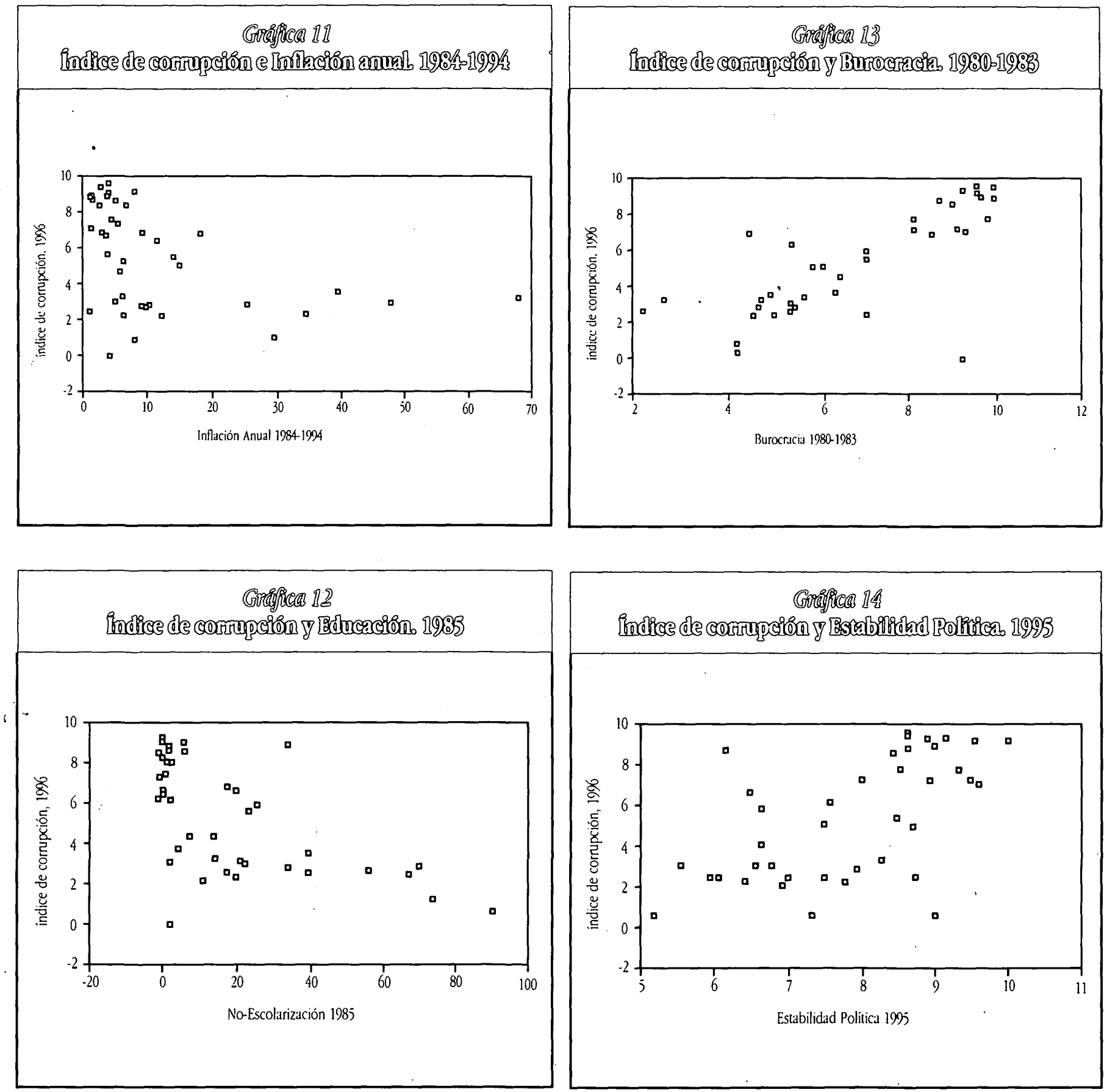

La inflación, contrariamente a lo esperado, no es estadísticamente significativa en el Modelo I. Según se esperaba, la Eficiencia Burocrática y la Estabilidad Política están muy correlacionadas. Por lo tanto, aislar el efecto único de cada variable individual es imposible.

Dado que ni las variables regionales ni la variable de la inflación incluidas en el Modelo I eran estadísticamente significativas, centraré mi argumentación en las variables estadísticamente significativas: Libertad Económica, Eficiencia Burocrática, No-Escolarización, Estabilidad Política y el PIB. La Tabla 2 muestra

que la burocracia, la libertad económica, la no-escolarización y el índice de crecimiento del PIB son estadísticamente significativos a partir del diez por ciento o más.

A medida que se incrementa en uno la eficiencia burocrática, el nivel de corrupción decrece en un $.544^{3}$. Los altos niveles de exceso de burocracia en las organizaciones burocráticas, junto con un sistema judicial ineficiente, ocasionan retrasos en el sistema. Por lo tanto, produce, por un lado, ineficiencia para realizar las gestiones por los medios adecuados y por otra parte, obstáculıs para denunciar legalmente los casos en los que se 
Tablo ?

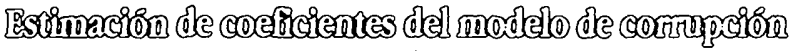

Mordelo II

\begin{tabular}{|c|c|c|c|c|c|}
\hline & B & $\begin{array}{c}\text { ERROR } \\
\text { ESTADÍSTICO }\end{array}$ & BETA & $t$ & SIG. \\
\hline Eficiencia Burocrática & .544 & .217 & .436 & 2.503 & 0.18 \\
\hline Libertad Económica & -8.51 & .510 & -.217 & -1.668 & .105 \\
\hline No-Escolarización & $-3.3 \mathrm{E}-02$ & .015 & -.267 & -2.185 & .037 \\
\hline OECD & .609 & .916 & -.107 & -.665 & .511 \\
\hline Estabilidad Politica. 1995 & .210 & 3.43 & .092 & .612 & .545 \\
\hline $\begin{array}{l}\text { Índice de Crecimiento } \\
\text { del PIB. 1983-1993 }\end{array}$ & .284 & .170 & .204 & 1.666 & .106 \\
\hline Inflación Anual. 1984-1994 & $-1.6 \mathrm{E}-02$ & .024 & -.078 & -.663 & .513 \\
\hline Asia Oriental & -.980 & .1027 & -.133 & .954 & .347 \\
\hline $\mathrm{R} 2$ & .732 & & & & . \\
\hline F-Estadística & $\begin{array}{l}10.587 \\
(\text { sig. 000) }\end{array}$ & & & & \\
\hline
\end{tabular}

utilizan procedimientos ilegales. A corto plazo, la ineficiencia produce nepotismo y, a largo plazo, comupción. Así, donde el retraso produce comupción, la ineficiencia se valorará positivamente, y donde el servicio corrupto es ilegal, la falta de escrúpulos será una gran recomendación del sistema burocrático para facilitar la corrupción (ACKERMAN, 1996).

Tal como se esperaba, a medida que se incrementa en uno la libertad económica, el nivel de corrupción disminuye en un .851. El Índice de Libertad Económica mide cómo de bien puntúan estos países en una lista de diez factores económicos. A mayor puntuación, mayor interferencia del gobierno en la economía y, por lo tanto, menor libertad económica y más corrupción. Los funcionarios públicos y los políticos son más propensos a abusar y manipular las tendencias de los negocios en pequeñas economías dominadas por una cuantas empresas.

Según muestra la Tabla 2, a medida que el índice de PIB, que capta el nivel de desarrollo económico en un país, entre 1983 y 1993 aumenta, disminuye la corrupción. A medida que aumenta un punto porcentual el índice de crecimiento del PIB, el nivel de corrupción disminuye en un 284 .

El siguiente determinante hipotético de la corrupción en el modelo conceptual era la igualdad social. La educación se usó como un equivalente de la igualdad social en el modelo. Se mi- dió la educación por el porcentaje de población que no estaba escolarizada en 1985. Según lo esperado, a medida que aumenta el número de no-escolarizados en un punto porcentual, la corrupción aumenta en un 0.033. Esto sugiere que la baja igualdad social está asociada a una corrupción elevada.

El R2 ajustado en el modelo es .732. Esto significa que el $73.2 \%$ de la variación en la corrupción se explica por las variables aquí descritas en el Modelo I. Sin embargo, dada la fuerte asociación entre la eficiencia burocrática y la mencionada estabilidad políica, se especificó y presentó un segundo modelo para consignar la posibilidad de una estricta multicolinearidad. Una de las variables que está fuertemente correlacionada con la eficiencia burocrática (la Estabilidad Política) está excluida. La medición también se abandona en el segundo modelo. El Modelo II, en la Tabla 2, muestra la relación entre la variable dependiente (el índice de comupción de 1996), y el bloque de variables independientes que fueron estadisticamente signifi. cativas en el Modelo I, excluyendo la estabilidad política y la medición de la OCDE.

En el Modelo II, a medida que aumenta en uno la eficiencia burocrática, el nivel de corrupción disminuye en un .594. A medida que la libertad económica aumenta en uno, el nivel de comupción disminuye en un .798. A medida que la no-escolarización se incrementa en un punto porcentual, la corrupción aumenta 


\begin{tabular}{|c|c|c|c|c|c|}
\hline \multicolumn{6}{|c|}{ 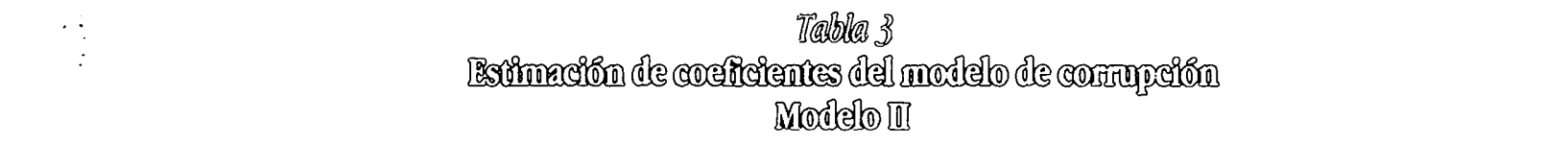 } \\
\hline & B & $\begin{array}{c}\text { ERROR } \\
\text { ESTADÍSTICO }\end{array}$ & BETA & $t$ & SIG \\
\hline Eficiencia Burocrática & .594 & .173 & .475 & 3.441 & .002 \\
\hline Libertad Económica & .798 & .484 & -.203 & .1648 & .10 \\
\hline No-Escolarización. 1985 & $-2.9 \mathrm{E}-032$ & .014 & -241 & -2.109 & .043 \\
\hline $\begin{array}{l}\text { Índice de Creciniento } \\
\text { del PIB. 1983-1993 }\end{array}$ & .265 & .164 & .191 & 1.612 & .117 \\
\hline Inflación Anual. 1984-1994 & $-9.6 \mathrm{E}-03$ & .022 & -0.48 & -.445 & .659 \\
\hline Asia Oriental & .634 & .902 & -.086 & -.703 & .487 \\
\hline R2 & .727 & & & & \\
\hline F-Estadística & $\begin{array}{c}14.64 \\
\text { (sig. } 000)\end{array}$ & & & & \\
\hline
\end{tabular}

en una 0.002. Estos resultados son compatibles con los hallazgos del Modelo I, la única diferencia estriba en las magnitudes.

El R2 ajustado en el modelo es .677. Esto significa que el $67.7 \%$ de la variación en la corrupción se explica por las variables aquí descritas en este modelo, corregidas por el número de variables explicativas usadas en el presente modelo. El R2 en el Modelo II es menor que en el Modelo I, pero esto era de esperar, dado que hay menos variables explicativas en el Modelo II.

Finalmente, se calcularon las elasticidades de las variables significativas para poder observar el porcentaje de corrupción explicado por las variables explicativas aquí expuestas. Según muestra la Tabla 4, de un aumento del uno por ciento en la media de la libertad económica, el índice de corrupción disminuye en un $0.43 \%$ en el Modelo I. En el Modelo II, el índice de corrupción disminuye en aproximadamente un $0.40 \%$. Para un aumento del uno por ciento en la eficiencia burocrática, el índice de corrupción se incrementa en un .32\% en el Modelo I, mientras que en el Modelo II se incrementa en un .34\%. Con respecto al PIB, un incremento del uno por ciento en el índice de crecimiento del PIB, incrementa el índice de corrupción en un $0.53 \%$ en el Modelo I, mientras que en el Modelo II, aumenta en un $0.49 \%$. La elasticidad calculada para la variable de NoEscolarización muestra que un aumento del uno por ciento en la no-escolarización, disminuye el índice de corrupción en un $0.07 \%$ en el Modelo I, mientras que en el Modelo II el decremento es del $6 \%$. La mayor elasticidad, en valor absoluto, es la de la Estabilidad Política, que equivale a $0.61 \%$. Por lo tanto, se- gún el primer modelo, una política orientada a combatir la corrupción, debería empezar por crear un ambiente de estabilidad política en un país. El segundo modelo muestra que una política orientada a combatir la corrupción debería tener como primer objetivo un aumento del índice de Crecimiento del PIB en un país. En ambos modelos, la educación parece ser la menos responsable en comparación con otras variables. Podría deberse al hecho de que la educación es una medida efectiva para combatir la corrupción a largo plazo.

\begin{tabular}{|c|c|c|}
\hline \multicolumn{3}{|c|}{ 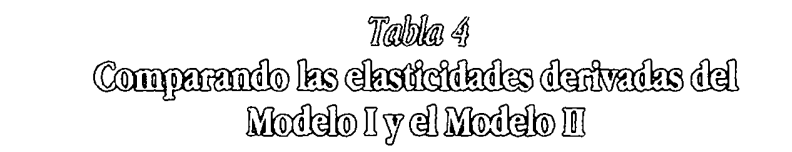 } \\
\hline & $\begin{array}{l}\text { ELASTICIDADES } \\
\text { MODELO I }\end{array}$ & $\begin{array}{l}\text { ELASTICIDADES } \\
\text { MODELO II }\end{array}$ \\
\hline Libertad Económica & -.043 & -0.40 \\
\hline $\begin{array}{l}\text { Índice de Crecimeinto } \\
\text { del PIB }\end{array}$ & 0.53 & 0.49 \\
\hline No-Exscularización & -0.07 & -0.06 \\
\hline Eficiencia Burocrática & 0.32 & 0.34 \\
\hline Estabilidad Politica & 0.61 & \\
\hline
\end{tabular}




\section{Conclusión}

Los datos disponibles sobre corrupción han permitido la posibilidad de explorar los determinantes de la corrupción desde una perspectiva empírica. Esto es esencial para convertir la teoría fértil en recomendaciones políticas prácticas. Aunque los resultados obtenidos hasta ahora son un estudio inicial y, por tanto deberían tratarse con precaución, los datos sugieren que existe una relación negativa entre la corrupción y la libertad económica, el crecimiento del PIB, la eficiencia burocrática y la educación. La evidencia también sugiere que la corrupción no es más severa en países pertenecientes a Asia oriental, ni menos severa en la OCDE, sino que es una red de factores controlados.

Las contribuciones de estos resultados representan un paso adelante en el diseño e implementación de cualquier política pública. Las burocracias obstaculizadoras asociadas unidas a los bajos .niveles de libertad económica y el bajo nivel de educación, permiten que aflore la corrupción en el sector público.

Cuando los ciudadanos perciben niveles generalizados de corrupción en el sector público, se dan cuenta de que los que se supone tenían que defender el Estado de Derecho son, de hecho, los que lo violan. En este contexto, un programa de bonos de comida, más que beneficiar a la gente pobre, podría diseñarse para beneficiar a los productores de comida y a los funcionarios públicos. Bajo estas circunstancias, la voluntad popular nunca aceptaría nuevos programas de gastos. Por ejemplo, intentos del gobierno de aumentar la recaudación de impuestos para la implementación de un programa social, sería imposible. Los ciudadanos podrín posiblemente creer que la recaudación de impuestos sería utilizada para enriquecer a los funcionarios públicos y, por lo tanto, los ciudadanos se resistirín a la implantación de esta política pública no pagando impuestos.

Este estudio sugiere las siguientes recomendaciones y estrategias parã reducir la compción. Ia experiencia indica que debe intentarse una combinación de las estrategias. Sin embargo, el tipo y la naturaleza de la combinación dependerá de las condiciones que prevalezcan en cada país.

\section{Recomendaciones y estrategias:}

- Creación de una política orientada a la implantación de un sistema político estable, donde puedan llevarse a cabo las reformas económicas, judiciales y de democratización institucional.

- La creación de políticas económicas orientadas a aumentar el Producto Interior Bruto en un país.

- La creación de incentivos para aumentar los niveles de libertad económica y disminuir la interferencia del gobierno en la economía.

- La creación de unas estructuras burocráticas fuertes y eficientes basadas en cristerios de imparcialidad y la competencia.

- La creación de una política orientada a animar a los sistemas educativos basados en la moral y la ética cívica.

Un paso siguiente razonable para analizar los problemas en el diseño e implementación de una políica pública sería extender el estudio de las causas de la corrupción entre los distintos países para conseguir mejores controles internos y externos en el gobierno. 


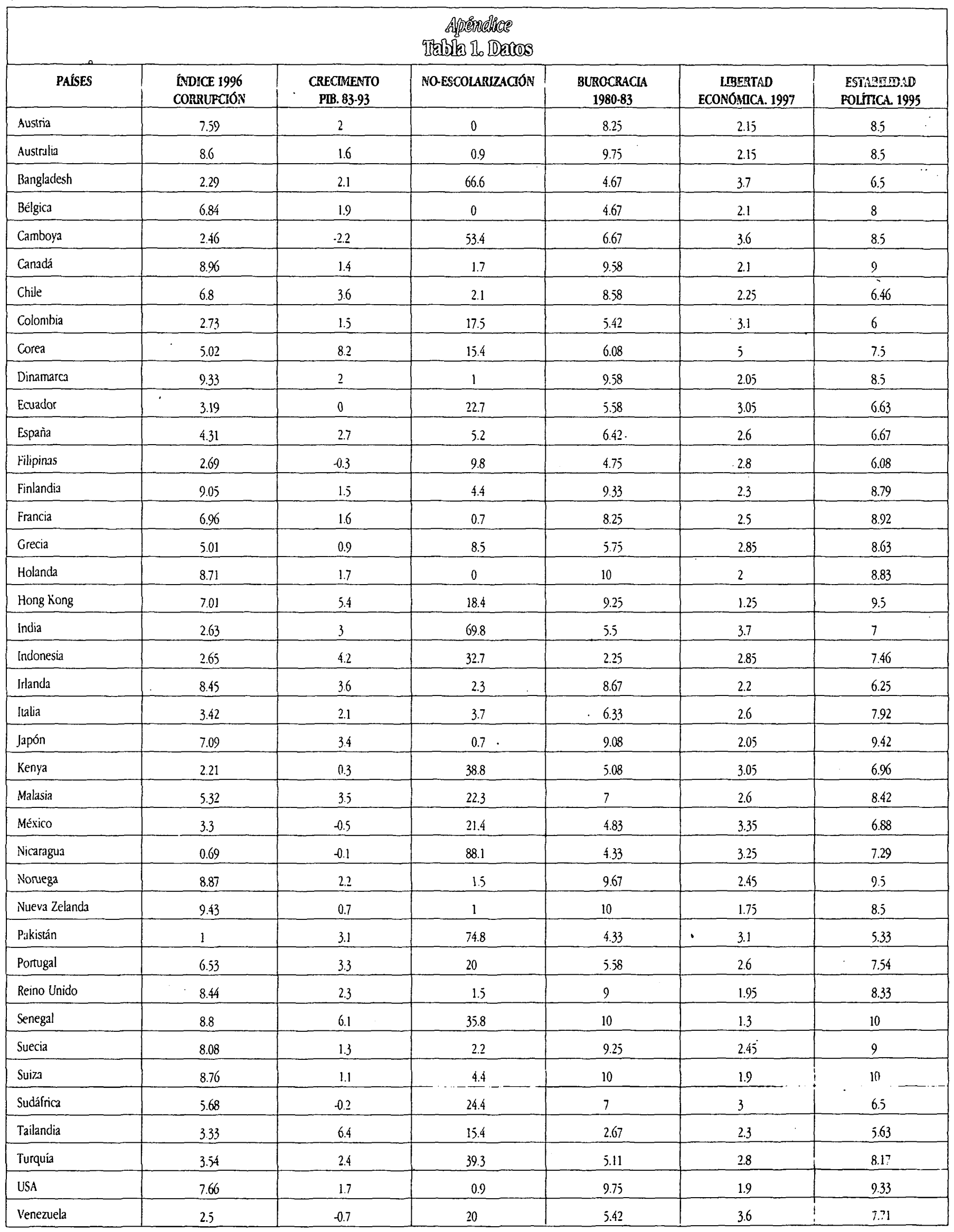




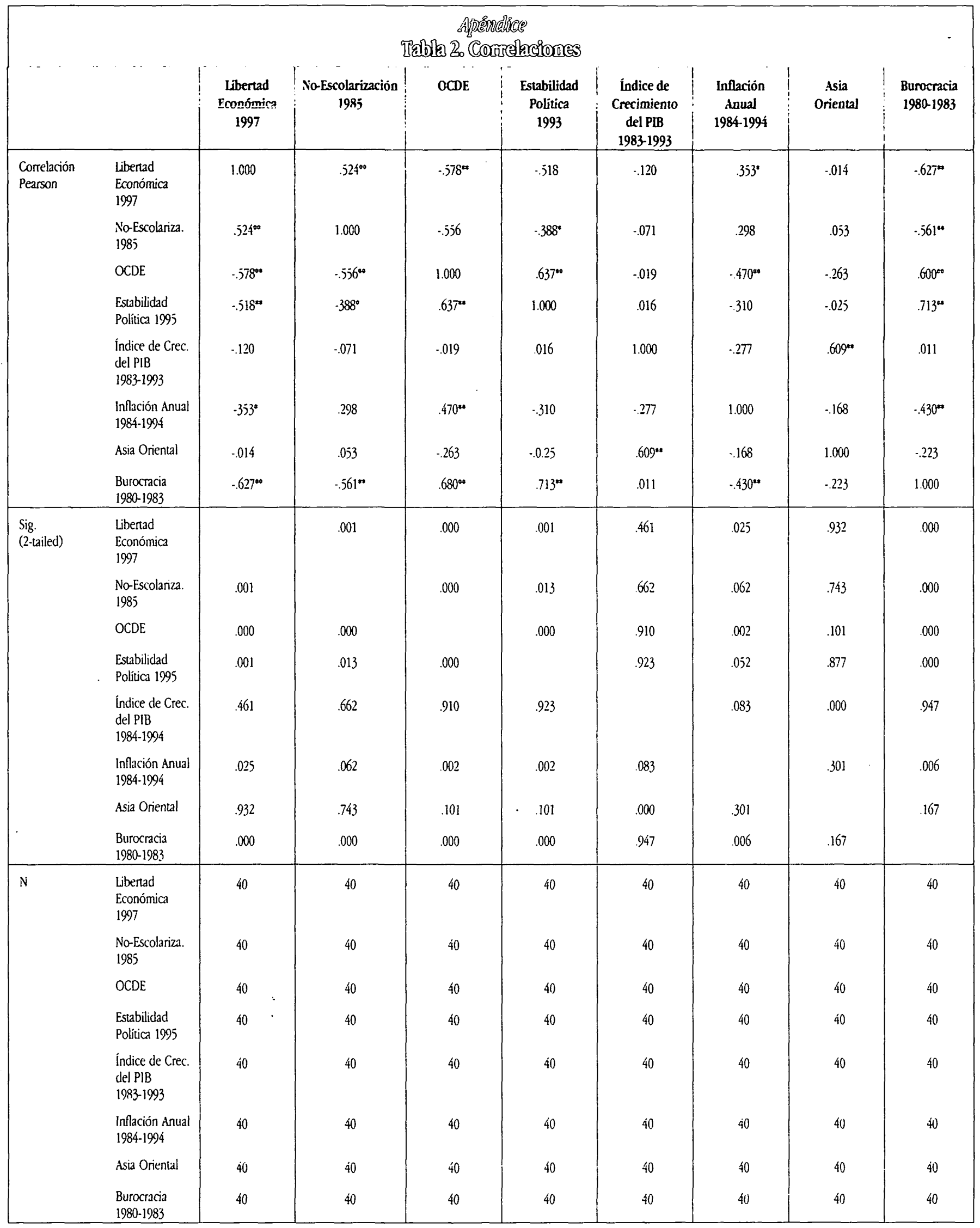

* La currelación es significante al nivel de 0.01 (2-tailcd).

- La correlación es significante al nivel de 0.05 (2-tailed). 
Traducción de Laura ALBA MUNoz.

Revisión Gemma SÁNCHEz RONTOMÉ.

- Georgetown Public Policy Institute.

' Entre otros, Susan Rose Ackerman (1978), Mauro (1995) y Tuluock (1990).
' Los Factores Económicos puntuados son: Política Comercial; Política Fiscal; Intervención del Gobierno en la Economía; Políica Monetaria; Flujos de Capial e Inversión Extranjera; Políica Bancaria; Controles Salariales y del Precio; Derecho de Propiedad; Regulación y el Mercado Negro. (The Heritage Foundation, 1997)

"Un alto nivel del índice de comupción, indica un bajo nivel de compción.

\section{Bibliografia}

ACKERMAN, Susan Rose (1996), . When is Comuption Harmful, World Development Report. Borrador, 1 de Agosto.

_ (1978), Comuption: A Study in Political Economy, New York Academic Press.

ADES, Alberto y Di TeLLA, Rafael (1994), •A Competition and Comption. Applied Economics Discussion Paper Series Number 169. Oxford: Oxford University.

Bardhan, Pranab.(1996), The Economics of Comuption in Less Developed Countries: A Review of Issues. Trabajo inédito. University of California, febrero.

BhaGwan, Jagdish (1982), Directly Unproductive, Profit-Seeking (DUP) Activities. Journal of Political Economy. 90, n. ${ }^{9}$.

Clements; Benedict; Rejane; Hugounenq y SChWartz, Gerd (1995), Govemment Subsidies: Concepts, Intemational Trends and Reform Options. IMF Working Papers 95/91. Washington D.C.; Intemational Monetary Fund.

Evan, Peters (1996), •Bureaucratic Structures and Economic Performances in Less Developed Countries. Trabajo inédito. University of California. Febrero.

Departamento de Hacienda (1995), Unproductive Public Expenditures: A Pragmatic Approach to Policy Analysis. Pamphlet Series 48. Washington D.C. Fondo Monetario Intemacional.

GoulD, David J. y AMARo ReYES, José A. (1983), Bureaucratic Comuption and Underdevelopment in the Third World. Nueva York: Pergamon.Press.

HOLMES; JOHNSON y KIRCKPATRICK (1997), 1997 Index of Economic Freedom. Washington D.C.: Heritage Foundation and The Wall Street Journal. Febrero.

HeIDenheimer, Amold J.; Johnston, Michael y LE VINE, Victor (eds.) (1989), Political Comuption: A Handbook New Brunswick, NJ: Transaction Books.

HONGYI Li; LyN SQUTRE y HENG-Fu ZOU (1996), Explaining International and Intertemporal Variations In Income Inequality. Trabajo inédito. World Bank.

HuNtINGTON, S.P. (1983), The Third Wave: Democratization in the Late Twentieth Century. Norman: University of Oklahoma Press.
POPE, Jeremy (ed.) (1996), The T7 Source Book Transparency International. Berlin, Alemania.

JOHnston, Michael (1986), -The Political Consequences of Cornuption: A Reassessment. Comparative Politics Review. Vol. 35. Julio.

KRUEGER, E. (1974), The Political Economy of a Rent-Seeking Society. American Economics Review. Vol. 64, n.. 3, pp. 291-303.

MaURo, Paulo (1996), The effects of Comuption-on Growth, Investment, and Government Expendirure. IMF Working Paper. Policy Development and Review.

_(1995), Cornption and Growth. Quarterly Joumal of Economics. Vol. 110, n. 3. Agosto.

SAHY, Raaj K. (1996), Social Osmosis and Patterns of Crime. Joumal of Political Economy. 99 , pp. 272-295.

SACHS, Jeffrey y WaRNER, Andrew (1995), .Natural Resources Abundance and Economic Growth. NBER Working Papers 5398. Cambridge, MA: National Bureau of Economic Research.

SCHLEIFER, A. y VISHMY, R. (1993), .Comuption. Quarterly Joumal of Economics. Vol. 109, pp. 599-617.

TULLOCK, Gordon (1990), -The Costs of Special Privilege, en JAMES, E. Alt y KENNETH, A. Shepsle (eds.), Perspectives on Positive Political Economy. Cambridge: Cambridge University Press.

TOUCKER, J. (1996), ·Comption Index. Financial Times, 3 de Junio.

Palmer, H. (1995), •A Global Gauge of Greased Palms. The New York Times, domingo, 20 de Agosto.

World Bank (1996), .From Plan to Market. World Detelopment Report 1996. Washington D.C.: Oxford University Press. 